Confirmed $\mathrm{CRC}=333, \mathrm{PPV}=95.14 \%$

Adenomas $=12(3.43 \%): 7$ required surgery, 1 EMR

Benign lesions $=5(1.43 \%): 2$ required surgery

223 of $350(63.71 \%)$ informed of CRC: 219 had CRC, 4 had adenomas

102 (29.14\%) informed of "lesion": 90 had CRC, 12 had benign disease

25 (7.14\%) no record (of discussion with patient): 24 had CRC, 1 had adenoma

Consultant colonoscopists (241 records) PPV 95.44\%

166 out of 241 (68.18\%) informed of CRC: $163 \mathrm{had}$ CRC, 3 had adenoma

58 (24.07\%) informed of "lesion": 50 had CRC, 3 had adenoma, 5 had benign disease

17 (7.05\%) no record: 17 had CRC

Trainee colonoscopists (81 records) PPV 92.59\%

47 out of 81 (58.02\%) informed of CRC: 46 had CRC, 1 had adenoma

$26(32.10 \%)$ informed of "lesion": 22 had cancer, 4 had adenoma

$8(9.88 \%)$ no record: 7 had CRC, 1 had adenoma

Nurse colonoscopists (28 records) PPV 100\%

10 out of 28 (35.71\%) informed of CRC, 18 out of 28 $(64.29 \%)$ informed of "lesion"

Conclusion This data shows that colonoscopists are proficient at diagnosing CRC (PPV 95.14\%). Those cases not confirmed with CRC usually have serious pathology which often requires surgery (9 out of 17). Yet only $63.71 \%$ of patients were informed of CRC. Consultants informed $68.18 \%$, trainees informed $58.02 \%$ and nurses informed only $35.71 \%$.

To reduce delay in CRC treatment and to give patients more time to deal with CRC diagnosis, colonoscopists should inform patients of a suspicion of CRC (and not a "lesion") and record this on reports.

Disclosure of Interest None Declared.

\section{PWE-003 VARIATIONS IN ADENOMA DETECTION RATE AND CANCER DETECTION RATE IN INDIVIDUALS FROM DIFFERENT ETHNIC GROUPS UNDERGOING BOWEL CANCER SCREENING COLONOSCOPY}

${ }^{1}$ A. M. Verma*, ${ }^{2}$ V. Lewin, ${ }^{1}$ A. P. Chilton, ${ }^{3}$ J. de Caestecker, ${ }^{1}$ A. Dixon, ${ }^{4}$ J. Jameson, ${ }^{3}$ P. Wurm, ${ }^{3}$ R. J. Robinson. 'Gastroenterology, Kettering General Hospital NHS Foundation Trust, Kettering; ${ }^{2}$ Endoscopy, Kettering General Hospital NHS Foundation Trust, Kettering; ${ }^{3}$ Gastroenterology, University Hospitals Leicester NHS trust, Leicester, UK; ${ }^{4}$ Endoscopy, University Hospitals Leicester NHS trust, Leicester, UK

\subsection{6/gutjnl-2014-307263.263}

Introduction The prevalent round of the Bowel Cancer Screening Programme (BCSP) in England commenced in August 2006. Analysis of the first three years of the BCSP reveals a mean adenoma detection rate (ADR) of 46.5\% (range 21.9-59.8\%), and a mean polyp detection rate (PDR) of 59.7\% (range 39.8$76.3 \%)^{1}$

Anecdotally, BCSP colonoscopists have suggested that the PDR, ADR and cancer detection rates in screened individuals of South Asian descent may be lower than those of Caucasian (white) descent. This has never been proven as the BCSP does not record ethnic origin of screened individuals.

Methods Between May $1^{\text {st }}$ and December $31^{\text {st }} 2013$, every screened individual in Leicester and Kettering had their selfselected ethnic origin recorded in a database. The endoscopic findings and histology results noted in the Exeter online database

\begin{tabular}{llll}
\multicolumn{4}{l}{ Abstract PWE-003 Table 1 } \\
\hline & "White" & "Asian" or "Asian British" & P value \\
\hline Cancer detection & $6.13 \%$ & $0.99 \%$ & $<0.08$ \\
PDR & $57.36 \%$ & $48.09 \%$ & $<0.002$ \\
ADR & $35.64 \%$ & $31.68 \%$ & $<0.02$ \\
\hline
\end{tabular}

was correlated to the database containing ethnic origin data and analysed.

Results 851 screened individuals (colonoscopy), 466 individuals had polyps (394 adenomas), $\mathrm{PDR}=54.76 \%$, ADR $=46.30 \%$, cancer detection rate $=5.41 \%$.

734 "White" individuals $(86.25 \%)$

45 individuals had cancer (cancer detection rate $=6.13 \%$ )

421 individuals had polyps, PDR $=57.36 \%$ (95\% CI: $53.75-$ $60.89 \%)$

353 individuals had polyps, ADR $=48.09 \%$ (95\% CI: 44.50 $51.71 \%)$

101 "Asian or Asian British" (11.87\%)

1 individual had cancer (cancer detection rate $=0.99 \%$ )

36 individuals had polyps, PDR $=35.64 \%$ (95\% CI: $26.99-$ $45.35 \%)$

32 individuals had polyps, ADR $=31.68 \%$ (95\% CI: $23.42-$ 41.29\%)

16 "Mixed", "Black or Black British" or "Other Ethnic Groups" $(1.88 \%)$

0 cancers, 8 individuals with polyps/adenomas $(\mathrm{PDR} / \mathrm{ADR}=$ $50 \%)$

Too few to meaningfully analyse

Conclusion This analysis reveals a statistically significant lower ADR and PDR for South Asian screened individuals when compared to Caucasian (White) individuals. There is also a strong trend showing a lower cancer detection rate. This is important for clinicians to be aware of so that they can fully inform individuals undergoing colonoscopic screening.

For regions with large South Asian populations, this observation can be used to appropriately plan services. ADR and cancer detection rates in these regions may be lower and may be a factor in the regional variations of ADR and cancer detection across the BCSP.

\section{REFERENCE}

1 Lee TJW, Rutter MD, Blanks RG, et al. Colonoscopy quality measures: experience from the NHS Bowel Cancer Screening Programme. Gut 2012;61:1050-1057 doi:10.1136/gutjnl-2011-300651

Disclosure of Interest None Declared.

\section{PWE-004 EXTRACOLONIC FINDINGS ON CT COLONOGRAPHY}

A Ashcroft*, S Jawad, P Mahendran, R Greenhalgh, A Poullis. St. George's Hospital, London, UK

\subsection{6/gutjnl-2014-307263.264}

Introduction Colonoscopy is often the first line investigation for detection of lesions within the large bowel and remains the gold standard in investigating for suspected colon cancer. However if endoscopy is either incomplete, determined too hazardous or declined computed tomographic colongraphy is the next investigation of choice. ${ }^{1}$ One of the additional and potentially beneficial features of CT is in the detection of extracolonic lesions, with studies suggesting approximately $40 \%$ of scans reveal one or more extracolonic abnormality including 14\% detecting 'significant findings' requiring further investigation. ${ }^{2}$ 
Methods We performed a retrospective analysis of all patients undergoing CT imaging of the large bowel, both CT colonography or plain abdominal CT (if CT colonography was not possible) at one south London hospital in a 13 month period between 2012-2013. Any extracolonic findings were determined either significant, where requiring further investigation or treatment, or insignificant by the reviewer. Any subsequent outcome of the significant findings was also sought.

Results A total of 257 scans were reviewed comprising of 250 (97\%) CT colonography and 7 (3\%) plain abdominal CTs in 104 (40\%) male and $153(60 \%)$ female patients. The average age was 68 years (range 39-91). A total of 163 (63\%) of scans detected at least one extracolonic finding, with 55 (21\%) of these significant. Sites included 13 liver, 7 lung, 6 pancreatic, 5 renal and 5 adrenal. Further investigation based on these findings revealed $5(1.9 \%)$ malignancies. One patient was found to have a renal cell carcinoma and went on to have curative surgery. One patient was diagnosed with pancreatic cancer and one with hepatocellular carcinoma, both of which were managed palliatively, and one patient was found to have peritoneal recurrence of a previously treated colonic adenocarcinoma. One scan discovered lung and liver metastases along with the causative colonic primary. Other notable findings included a $5.3 \mathrm{~cm}$ AAA and a pulmonary embolus seen in a segmental lower lobe pulmonary artery. There were a 209 insignificant findings in 139 $(54 \%)$ of the CTs, with a maximum of 5 in a single scan.

Conclusion This study helps to highlight the potential additional benefit of CT colonography over endoscopic visualisation of the large bowel. The prevelance of extracolonic findings in this cohort was high, in keeping with previous studies, with CT colonography having value is its detection of extracolonic malignancies, staging and other serious conditions. However there was also a substantial rate of additional investigation for subsequently benign findings.

\section{REFERENCES}

1 Taylor SA et al. Guidelines for the use of imaging in the NHS Bowel Cancer Screening Programme: Second Edition. 2012

2 Xiong T et al. Br J Radiol 2005 Jan;78(925):22-9

Disclosure of Interest None Declared.

\section{PWE-005 MANAGEMENT OF HIGH RISK COLONIC POLYPS}

C Hayward, CR Chimakurthi* . Gastroenterology, Derriford Hospital, Plymouth, UK

\subsection{6/gutjnl-2014-307263.265}

Introduction Colorectal cancer is a significant health problem, the importance of which will increase substantially in the coming years. Demand for colonoscopy will increase and so will demand for complex polypectomy to deliver a reduction in incident rates.

Methods Colonoscopy reports with an endoscopic diagnosis "high risk colonic polyp" were examined over a 6 month period. Histology was reviewed to determine the precise histological classification of all polyps. Repeat procedures over the following 2 years were reviewed for completeness of initial resection. Complete adenoma clearance rates were calculated based on observation of residual polyps or residual polyp tissue at previous polypectomy site.

Results Twenty one colonoscopists performed 2139 colonoscopies. The median caecal intubation rate was $93 \%$. The number of procedures performed by individual endoscopists varied between 14-464. The median was 64 procedures. In 564 (24\%) cases, one or more than one polyp were identified. Individual endoscopist adenoma detection rates (ADR) did vary. The median ADR was 24\% (0-44\%). In 79 cases the endoscopic diagnosis was reported as "high risk". When the initial reports were analysed with histology, 52 (69 \%) cases met BSG high-risk criteria. Of the 52 high-risk polyp cases, histology confirmed adenocarcinoma in 10 cases. Surgery was performed for 5 benign cases. Of the 44 benign lesions managed endoscopically, 35 (80\%) patients were recommended to undergo a repeat procedure (s). In total 24 patients underwent one repeat procedure, 8 underwent 2 repeat procedures and 3 patients underwent 3 repeat procedures over the follow up period.

Complete adenoma clearance rate at index endoscopy in this audit was achieved in 11 (31\%) cases. Two further cases were regarded as having complete clearance following a subsequent resection.

Conclusion The finding of multiple or complex polyps puts pressure on colonoscopists. Difficult procedures may adversely affect ADR. Although key performance indicators such as caecal intubation rate have improved with national training programmes, this audit and other studies have demonstrated variation in therapeutic outcomes. ${ }^{1}$ Scoring systems for complex polypectomy should be employed to encourage endoscopists to defer polypectomy in some situations. ${ }^{2}$ Designated therapeutic lists will benefit patients and endoscopy units with reduction in repeated procedures and improved mentoring/training opportunities in complex polypectomy.

\section{REFERENCES}

1 Pohl H, Srivastava A, Bensen SP, Anderson P, Rothstein RI, Gordon SR, et al. Incomplete polyp resection during colonoscopy-results of the complete adenoma resection (CARE) study. Gastroenterology 2013;144(1):74-80 e1

2 Gupta S, Bassett P, Man R, Suzuki N, Vance ME, Thomas-Gibson S. Validation of a novel method for assessing competency in polypectomy. Gastrointestinal Endoscopy 2012;75(3):568-75

Disclosure of Interest None Declared.

\section{PWE-006 NICORANDIL USAGE IS ASSOCIATED WITH COMPLICATED DIVERTICULITIS}

A O'Brien, A Young, F Duthie*. Pathology, Southern General Hospital, Glasgow, UK

10.1136/gutjnl-2014-307263.266

Introduction Nicorandil is widely used in the treatment of ischaemic heart disease, but over the last ten years there has been a growing body of literature reporting the association between nicorandil and ulceration of the gastrointestinal tract. In the setting of diverticulosis/diverticulitis, any superimposed ulceration may lead to significant complications, but data on whether or not nicorandil contributes to this is scanty and limited to one study which only investigated intestinal fistulae. ${ }^{1}$ Our aim is to identify if any potential association between all forms of complicated diverticulitis and nicorandil exists.

Methods We reviewed 100 reports of colonic resections with diverticular disease received in a single institution over a 6 month period (from June 2013 to January 2014) and divided them into those with complicated diverticulitis (defined as active diverticulitis in combination with perforation, fistulation, abscess formation or structuring) ${ }^{2}$ and those with uncomplicated diverticulitis or uninflamed diverticulosis. The age, sex, surgical indication and use of nicorandil were recorded for both groups.

Results 51 patients had complicated diverticulitis and 45 had uncomplicated diverticulitis or diverticular disease (7 diverticulosis 